\title{
Complex Chronic Patients and Atrial Fibrillation: Association with Cognitive Deterioration and Heart Failure
}

Queralt-Tomas $\mathrm{ML}^{1}$, Panisello-Tafalla $\mathrm{A}^{2}$, González-Henares $\mathrm{A}^{3}$, Clua-Espuny JL ${ }^{4}$, Campo-Tamayo $\mathrm{W}^{5}$ Muria Subirats $\mathrm{E}^{6}$, Forcadell-Arenas $\mathrm{T}^{7}$ and Gil-Guillen $\mathrm{VF}^{8}$ on behalf of the Ebrictus Research Group

${ }^{1}$ Nursing, EAP Tortosa-2-Oest, Catalonian Health Institut, SAP Terres de l'Ebre, Health Department, Generalitat de Catalunya, CAP Xerta, Spain

${ }^{2}$ EAP Aldea-Camarles-Ampolla, Catalonian Health Institut, SAP Terres de l'Ebre, Health Department, Generalitat de Catalunya, CAP Aldea, 43520, Spain

${ }^{3}$ EAP-Camarles-Aldea-Ampolla, Catalonian Health Institut, SAP Terres de l'Ebre, Health Department, Generalitat de Catalunya, CAP Ampolla, 43895, Spain. Department of Research, ICS Terres de l'Ebre, Research Institute University Primary Care (IDIAP) Jordi Gol, Spain

${ }^{4}$ EAP Tortosa 1-Est, Catalonian Health Institute, SAP Terres de l'Ebre, Health Department, Generalitat de Catalunya, CAP Temple, 43500 Tortosa, Spain. Department of Research, ICS Terres de l'Ebre, Research Institute University Primary Care (IDIAP) Jordi Gol. University Miguel Hernández, Elx

${ }^{5}$ EAP Tortosa Est. Catalonian Health Institute, SAP Terres de l'Ebre, Health Department, Generalitat de Catalunya. Family and Community Specialty Resident. CAP Temple, 43500 Tortosa, Spain

${ }^{6}$ EAP Tortosa Est. Catalonian Health Institute, SAP Terres de l'Ebre, Health Department, Generalitat de Catalunya. Family and Community Specialty Resident. CAP Temple, 43500 Tortosa, Spain

${ }^{7}$ Catalonian Health Institut, SAP Terres de l'Ebre, Health Department, Generalitat de Catalunya, CAP Temple, 43500, Spain

${ }^{8}$ Universidad Miguel Hernández. Cátedra Medicina de Familia. 03202 Elche, Spain. Clinical Evidence Based Medicine and Emotional Department, Miguel Hernández University, Family and Community Specialty

${ }^{*}$ Corresponding author: Clua-Espuny JL, EAP- Tortosa est, Institut Català Salut, SAP Terres de l'Ebre, Health Department, Generalitat de Catalunya. Plaça Carrilet, s/núm. 43500 Tortosa, Spain, Tel: (34) 9775100 18, Fax: (34) 9774457 28, E-mail: jlclua@telefonica.net

Citation: Queralt-Tomas ML, Panisello-Tafalla A, González-Henares A, Clua-Espuny JL, Campo-Tamayo W, et al. (2017) Complex Chronic Patients and Atrial Fibrillation: Association with Cognitive Deterioration and Heart Failure. J Clin Exp Res Cardiol 3(1): 104

Received Date: February 27, 2017 Accepted Date: April 08, 2017 Published Date: April 10, 2017

\begin{abstract}
Background: In developed countries, around 3-5\% of the people could be identified as chronic complex patients, and they are increasingly at risk of atrial fibrillation (AF) and cognitive impairment. The main objective of this study was to present the current findings on the association between AF and cognitive impairment and mortality risk among chronic complex outpatients (CCP).

Methods: A multicenter and prospective cohort study of mortality incidence was carried out from 1 January 2013 to 30 September 2016 in a sample of 932 adult patients registered as CCP. To predict hazard ratios, mean survival time, and survival probabilities, a multivariate Cox regression was used.

Results: The overall mortality among the CCP with AF was 40.9\% (CI95\% 35.4-46.4) and 56.9\% if associated with cognitive impairment ( $\mathrm{p}$ 0.001). The long-term survival was not different between the groups of CCP with AF and without AF ( $\mathrm{p} 0.463$ ). In the survival analyses, the outcome-independent factors were Pfeiffer score (HR 1.07 CI95\% 1.005-1.146, p 0.036), Barthel score (HR 0.99 CI95\% 0.98-0.99, p 0.019), Charlson index (HR 1.17 CI95\% 1.03-1.33, p 0.015), and heart failure (HR 1.91 CI95\% 1.33-2.74, p <0.001). Cognitive impairment was found to increase the mortality by two-fold (relative risk: 1.69; CI95\%: 1.31-2.17). The results showed higher stroke incidence but fewer and poor quality $(\mathrm{c}-\mathrm{TTR}<60 \%)$ treatment with oral anticoagulants among those with cognitive impairment and a Barthel score $<60(\mathrm{p}<0.001)$.

Conclusion: Persons with AF should be investigated for the presence of cognitive impairment and heart failure. A nurse-led, multidisciplinary home-based intervention - using an AF algorithm - should be able to demonstrate favourable effects in patients with AF by the use of an integrated approach specifically directed to AF.
\end{abstract}


Keywords: Atrial fibrillation; Cognitive impairment; Chronic complex patient; Heart failure; Mortality

List of Abbreviations: AF: Atrial fibrillation; ADL: Activities of Daily Living; CCP: Chronic Complex Patient; HF: Heart Failure; HR: Hazard Risk; IDIAP: Primary Care Research Institute Jordi Gol I Gurina; NOACs: Novel Oral Anticoagulants; SD: Standard Deviation; c-TTR: Time in Therapeutic Range

\section{Introduction}

The demographic evolution in developed countries is characterised by the emergence of new and complex health needs due to chronic multimorbidity [1-3]. With progressive aging, disease chronicity becomes something common. As a result, from 3 to $4 \%$ of the population is composed of complex chronic patients, and one of their risks is the increase in the incidence of atrial fibrillation and/or cognitive deterioration [4-6]. It is expected that the number of individuals with atrial fibrillation will increase $150 \%$ in the next four decades, especially in individuals aged over 80 years [7]. This way, this disease will be one of the main health economic burdens in the European population.

In addition, the evidence suggests that 15 to $20 \%$ of the individuals aged over 65 years will suffer some degree of cognitive deterioration [8]. In 2015, the estimated number of individuals with dementia in the world was 47.47 millions, and its evolution was expected to be 75.63 million by 2030 and 135.46 millions by 2050 [9]. Therefore, the incidence of dementia will double every 5.9 years of age increase, i.e., from 3.1/1000 person-years at age 60-64 to 175/1000 person-years at age $\geq 95$ [10].

Thus, the proposals for the approach and treatment of the two disorders are of great relevance, because they generate enormous challenges in both health and social care due to the burden of morbimortality [11-13]. The main goal of the present study was to determine the association between atrial fibrillation and cognitive deterioration in a cohort of complex chronic patients.

\section{Methods}

We carried out a multicentre and prospective cohort study of mortality incidence from 1 January 2013 to 30 September 2016 among chronic complex outpatients (CCP) attending primary care teams in the Terres de l'Ebre health area in Catalonia (Spain). All people included were managed by the public health system in Catalonia. Registry information was collected from the government-run healthcare provider responsible for all inpatient care in the county. The overall number of CCP registered was 3,490. We included a randomized sample of 932 adult patients registered in the electronic health record of primary care as CCP from 1 January 2013 to 31 December 2014. Patients were excluded if they resided in a long-term institutional setting. Alpha risk=0.05; precision=0.03.

Patient outcome was followed until death or study end (30 September 2016) since date of report as CCP in the electronic health record. Data included demographics, and functional, comorbidity, cognitive, and social assessment and were collected directly from the Shared Individual Intervention Plan [Pla d'intervenció individualitzat compartit (PIIC)] written and managed by nursing service in primary care. In the PIIC, determinants related to the personal factors and the social and physical environment are described as well a tailored personal approach according the patient's preferences in case of hospital readmission or emergency use, and main caregiver. The report is updated automatically to ensure that relevant information is shared across the electronic health record. Currently, $82 \%$ of people registered as CCP have this basic information in their PIIC.

\section{Definitions}

Chronic complex patient (CCP): Those who meet at least four of the following criteria: Age ( $\geq 65$ years old), chronic comorbidities $(\geq 4)$, psychosocial disorders (cognitive impairment or psychological disorder with functional disability), geriatric conditions such as functional disability (Barthel score $<55$, living in assisted living, nursing home, or with in-home caregivers) or recurrent falls or fall risk, previous high healthcare use (two hospitalisations not programmed for exacerbation of chronic pathologies or three emergency department visits in the last year), number of active medications in the last six months ( $\geq 4$ active medications), and living alone or with a caregiver $\geq 75$ years old. The group also could include patients who do not meet these criteria, but are affected by other clearly complex conditions, such as schizophrenia or mental illnesses with behavioural disorders that make their management significantly more difficult [14].

Stroke: Individuals with current stroke diagnoses in their medical charts after inclusion in the study were considered to have experienced an incident stroke. A previous stroke was considered to be present if the medical charts included a stroke diagnosis or if the individual, a caregiver, or a relative reported the diagnosis and it was found on supporting information from medical charts or other assessments. Records of inpatient care after baseline assessment with the International Classification of Diseases ( $10^{\text {th }}$ version; ICD-10) code prefixes I60, I61, I63, and I64 and H34, I67, and G45 as well as subsequent diagnoses of stroke or transient ischemic attack were compiled for all participants. All other diagnoses were based on information from assessments conducted during home visits and from records from hospitals, general practitioners, and institutional care facilities. A physician comprehensively reviewed the digital medical charts of individuals.

There are problems in defining fall risks, as many studies fail to specify an operational definition, leaving room for interpretation. 
A fall is an unintentional event that results in the person coming to rest on the ground or another lower level (W19.9 code in the electronic health record). According to the WHO, a fall is defined as the result of any event that caused the patient to end up on the ground against his or her will [15]. We used 'the report clinical in the electronic health record that a person had falls risk or previous recurrent falls with or without any serious injury'. If a patient is thought to be at high fall risk by medical or nursing staff, allied health, or caregivers, such patients will be identified as a 'fall risk' in the PIIC. This might include mention of the patient's level of orientation and cognition, gait and balance, continence status, and number and types of prescribed medications, as well as the number of diagnoses.

\section{Variables}

Sex: female (0), male (1)

Age: $<80$ years old (1), $\geq 80$ years old (2)

Number of CCP criteria: $<4(0), \geq 4$ (1)

Charlson Comorbidity Index, short version [16]

Current medications were asked about during the home visit and confirmed in medical records. Polypharmacy (defined as five or more daily medications): $<5$ (0), 5-9 (1), and $\geq 10$ (2). Oral anticoagulants (acenocumarol or warfarin) and TTR $\geq 60 \%$ (1), if TTR $<60 \%$ (2) or novel oral anticoagulants (NOACs) (0). Antidepressants and/or sedating or other drugs affecting the neurologic system: male (1), female (2). If there was a diagnosis of atrial fibrillation (AF), CHA2DS2VASCHAS-BLED scores were included.

Recurrent falls or fall risk: no (0), yes (1)

Hypertension not controlled by therapy ( $\geq 160 / 90 \mathrm{mmHg}$ ): no (0), yes (1). Blood pressure was measured as an average of separated follow-up measurements in the last six months.

Alcohol abuse vs dependence: no (0), yes (1)

Presence of cognitive impairment [17]: A disease-specific diagnosis of cognitive impairment, without specification of sub-type or severity, was used and measured by the Pfeiffer test [2]: [0-2 errors] = intact intellectual functioning (1); [ $\geq 3$ errors] = mild to severe intellectual impairment (2).

Presence of disability: Barthel $[\geq 60$ (1) $<60$ (2)] or Rankin $[<4$ (1) 5(2)] scores were used to assess dependence in ADL.

Presence of heart failure: it's a condition registered in electronic health record (CIE9 codes I11, I13 and I50, and subcodes included) according the presence of clinical Framingham criteria for Congestive Heart Failure and guide reference $[1,18]$.

Sociofamiliar risk: score in Gijon scale 10-14 (1), $\geq 15$ (2) [19].

We conducted an intention-to-treat analysis. Patients were followed up from cohort entry as CCP until the first event occurrence (death), end of the study period, or lost to follow-up. Categorical variables were expressed as frequencies (percentage), and quantitative variables were expressed as mean (standard deviation [SD]) or median (interquartile range). Demographic data were summarized using mean and SD or median and quartiles for continuous variables and percentages for categorical data. Data analysis information extracted was the adjusted risk estimates and 95\% confidence intervals (CI), and all statistical tests were two-sided at the $5 \%$ significance level. Statistical tests of homogeneity were performed using Cochran's Chi-squared test for homogeneity (Q) and the percentage of total variation across studies attributable to heterogeneity (I2). Using univariate linear regression analysis with the medication count as a continuous outcome variable, we identified explanatory variables that had significant ( $\mathrm{p} \leq 0.05)$ univariate linear associations with medication count. Time-to-event analysis was performed using nonparametric methods like the Kaplan-Meier and log-rank test. Multivariate Cox proportional-hazards regression models were fitted, adjusting for the following baseline characteristics and confounding and predictive factors of each event: age, sex, Charlson index, and factors in CHA2DS2VASC and HAS-BLED scales and active treatments. To predict hazard ratios, mean survival time, and survival probabilities, we used a multivariate Cox regression. The variables were included in a multivariable Cox model to identify their influence on the mortality. The non-hemorrhagic group and non-stroke group were considered as the references in all analyses. The analyses were performed using IBM SPSS version 19.0

Ethical approval was granted by the Ethics Committee Research Institute Primary Care Jordi Gol i Gurina (IDIAP), Health Department, Generalitat de Cataluña.

\section{Results}

A total of 932 patients were included (52.3\% CI95\% 49.1-55.6, female) as CCP in the study. The baseline characteristics are showed in Table 1. A total of 325 patients (34.8\% CI95\% 31.7-37.9) were diagnosed with AF (46.5\% CI95\% 40.8-52.0, female), and 260 (27.6\%) suffered a stroke: $20.2 \%$ ischemic, $7.4 \%$ hemorrhagic. The overall stroke incidence was $24.9 / 1,000$ persons per year.

The patients with AF were on average older, a larger proportion was male and aged $\geq 80$ years, and they had higher prevalence of heart failure (HF), Barthel score, Charlson score, and incidence of mortality than those without AF (Figure 1). Average age was 83.9 years (CI95\% 82.8-84.2). The average number of CCP criteria was 3.95 (CI 95\% 3.85-4.02). The global mortality was $40.9 \%$ (CI95\% 35.4-46.4). In the survival analyses of risk for death, the outcome-independent factors were Pfeiffer score (HR 1.07 CI95\% 1.005-1.146, p 0.036), Barthel score (HR 0.99 CI95\% 0.98-0.99, p 0.019), Charlson index (HR 1.17 CI95\% 1.03-1.33, p 0.015), and HF (HR 1.91 CI95\% 1.33-2.74, $\mathrm{p}<0.001)$. 


\begin{tabular}{|c|c|c|c|}
\hline $\begin{array}{l}\text { CCP PEOPLE } \\
(\text { (N 932) }\end{array}$ & $\begin{array}{l}\text { WITHOUT AF } \\
\text { (n1 607) }\end{array}$ & $\begin{array}{l}\text { WITH AF } \\
\text { (n2 325) }\end{array}$ & $\mathbf{P}$ \\
\hline $\mathrm{N}(\%)$ & 607 & $325(34.87 \%)$ & \\
\hline Age (average $\pm \mathrm{SD}$ ) & $81.8 \pm 10.86$ & $83.9 \pm 7.51$ & 0.002 \\
\hline Percentage $>80$ year-old $n(\%)$ & $408(67.2 \%)$ & $247(76.0 \%)$ & 0.003 \\
\hline Women n (\%) & & $151(46.5 \%)$ & 0.005 \\
\hline $\mathrm{CCP}$ criteria number (average $\pm \mathrm{SD}$ ) & $3.81 \pm 1.21$ & $3.95 \pm 1.12$ & 0.076 \\
\hline Charlson score (average $\pm \mathrm{SD}$ ) & $2.44 \pm 1.38$ & $2.68 \pm 1.36$ & 0.014 \\
\hline Hypertension n (\%) & $500(82.4 \%)$ & $274(84.3 \%)$ & 0.256 \\
\hline Diabetes mellitus n (\%) & $345(56.8 \%)$ & $149(45.8 \%)$ & 0.001 \\
\hline Dyslipidemia n (\%) & $354(58.3 \%)$ & $167(51.4 \%)$ & 0.025 \\
\hline Active smoking $\mathrm{n}(\%)$ & $50(8.2 \%)$ & $22(6.8 \%)$ & 0.253 \\
\hline Alcoholism active $\mathrm{n}(\%)$ & $12(2.0 \%)$ & $11(3.4 \%)$ & 0.137 \\
\hline Ischaemic Heart Disease n (\%) & $120(19.8 \%)$ & $76(23.4 \%)$ & 0.114 \\
\hline $\begin{array}{l}\text { Peripheral arterial occlusive disease } \\
\mathrm{n}(\%)\end{array}$ & $98(16.1 \%)$ & $51(15.7 \%)$ & 0.468 \\
\hline Failure Heart n (\%) & $131(21.6 \%)$ & $172(52.9 \%)$ & $<0.001$ \\
\hline $\begin{array}{l}\text { Daily medications number } \\
\text { (average } \pm \mathrm{SD})\end{array}$ & $8.49 \pm 3.68$ & $9.58 \pm 3.31$ & $<0.001$ \\
\hline CHADSVASC score (average $\pm \mathrm{SD}$ ) & $5.31 \pm 1.85$ & $5.00 \pm 1.26$ & 0.344 \\
\hline Stroke risk/year (average $\pm \mathrm{SD}$ ) & $6.11 \pm 2.53$ & $6.55 \pm 2.41$ & 0.485 \\
\hline HAS-BLED (average \pm SD) & $3.38 \pm 1.20$ & $2.96 \pm 1.09$ & 0.147 \\
\hline $\begin{array}{l}\text { Major Bleeding rate \%/year } \\
\text { (average } \pm \mathrm{SD})\end{array}$ & $6.06 \pm 4.38$ & $4.89 \pm 3.57$ & 0.207 \\
\hline Oral Anticoagulant treatment n (\%) & $30(4.9 \%)$ & $240(73.9 \%)$ & $<0.001$ \\
\hline Labile INR (TTR < 60\%) & $53.16 \pm 28.4$ & $53.08 \pm 27.31$ & 0.988 \\
\hline Hypertension no controlled n (\%) & $79(13.0 \%)$ & $28(8.6 \%)$ & 0.026 \\
\hline Stroke before CCP register n (\%) & $123(20.3 \%)$ & $77(23.7 \%)$ & 0.129 \\
\hline $\begin{array}{l}\text { Stroke after CCP register } \mathrm{n}(\%) / 1000 \\
\text { people }\end{array}$ & $\begin{array}{c}41(6.8 \%) \\
0.675\end{array}$ & $\begin{array}{c}25(7.7 \%) \\
0.769\end{array}$ & 0.701 \\
\hline Cognitive Impairment n (\%) & $236(38.9 \%)$ & $102(31.4 \%)$ & 0.014 \\
\hline Pfeiffer Test Score (average \pm SD) & $3.34 \pm 3.47$ & $2.54 \pm 2.80$ & $<0.001$ \\
\hline Barthel score (average $\pm \mathrm{SD}$ ) & $63.91 \pm 33.63$ & $70.2 \pm 28.09$ & 0.004 \\
\hline $\begin{array}{l}\text { Percentage Barthel score }<60 \text { Moderate } \\
\text { dependence } \mathrm{n}(\%)\end{array}$ & $241(39.7 \%)$ & $102(31.4 \%)$ & 0.007 \\
\hline Gijon score (average $\pm \mathrm{SD}$ ) & $10.28 \pm 4.86$ & $9.35 \pm 4.16$ & 0.377 \\
\hline Platelet antiaggregants n (\%) & $306(50.4 \%)$ & $79(24.3 \%)$ & $<0.001$ \\
\hline Statines treatment n (\%) & $24(30.8 \%)$ & $23(35.4 \%)$ & 0.342 \\
\hline Inhibitor proton pump $\mathrm{n}(\%)$ & $394(64.9 \%)$ & $249(76.6 \%)$ & $<0.001$ \\
\hline $\begin{array}{l}\text { Antidepressants and/or sedating or } \\
\text { similar } \mathrm{n}(\%)\end{array}$ & $346(57.6 \%)$ & $168(51.7 \%)$ & 0.069 \\
\hline Falls risk report n (\%) & $134(22.1 \%)$ & $54(16.6 \%)$ & 0.028 \\
\hline Death n (\%) & $186(30.6 \%)$ & $133(40.9 \%)$ & $0, .001$ \\
\hline $\begin{array}{l}\text { Average survival time (days) } \\
\text { (average } \pm S D)\end{array}$ & $971.71 \pm 1462.9$ & $1066.01 \pm 2455.4$ & 0.463 \\
\hline
\end{tabular}

Table 1: Baseline characteristics of Complex and Chronic Patients with Atrial Fibrillation 


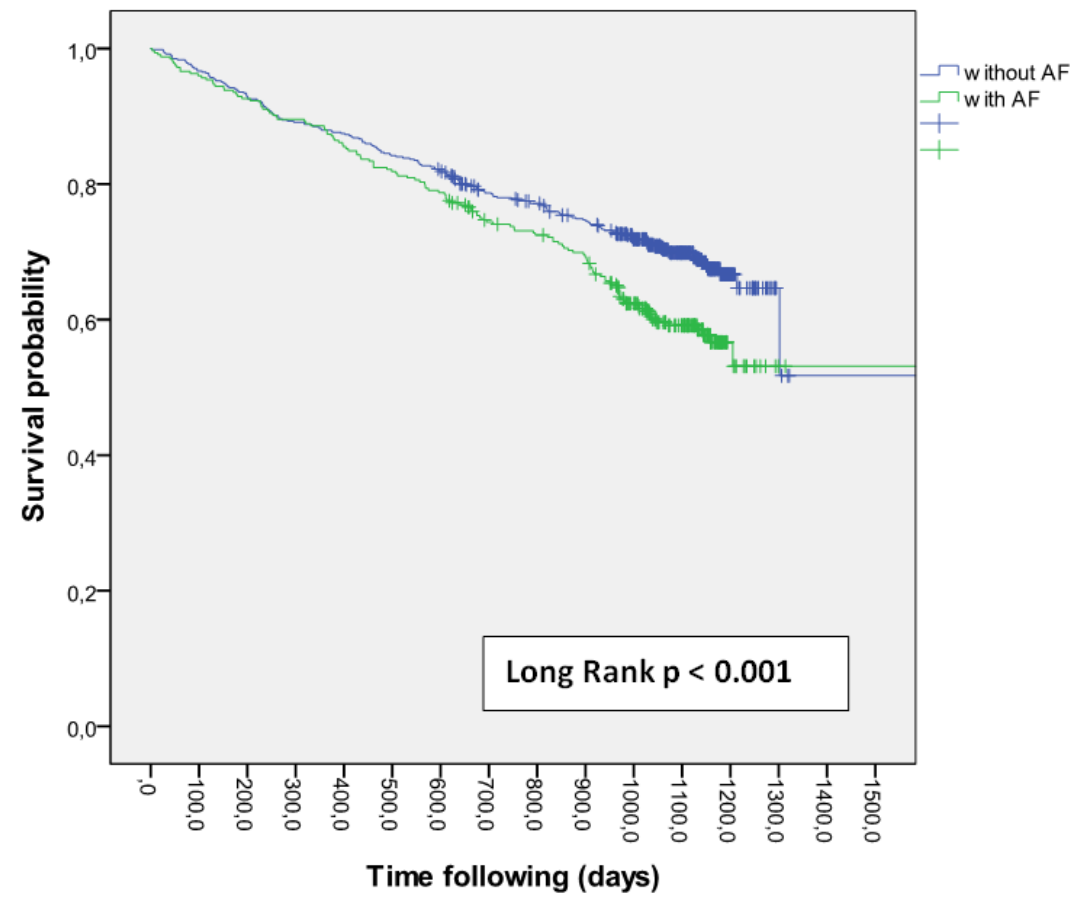

Figure 1: Survival among PCC people: with AF vs. without AF

A total of 102 patients (31.38 CI95\% 26.1-36.5) with AF had been diagnosed with cognitive impairment by the Pfeiffer test. The basal characteristics are showed in Table 2. The patients were more likely to be women (54.9\% CI95\% 44.7-65.0, p 0.008), be of older age $(86.45 \pm$ SD 5.90 vs $82.86 \pm$ SD7.90, p $<0.001)$, and have more CCP criteria $(4.63 \pm$ SD 1.19 vs $3.64 \pm$ SD 0.94 , p <0.001), a higher score in the Charlson index ( $3.24 \pm$ SD 1.40 vs $2.42 \pm$ SD $1.25, \mathrm{p}<0.001)$, a higher baseline burden of functional dependence in daily activities (Barthel index [53.15 \pm SD28 vs 77.9 \pm SD24.08, $<<0.001]$ ), and higher mortality (56.9\% vs 33.6\%, p < 0.001) (Figure 2). Cognitive impairment was found to increase the mortality by two times (relative risk: 1.69; CI95\% 1.31-2.17). Other interesting points were that patients with cognitive impairment were more likely to be treated with central nervous system medications (antidepressants, sedating, and others) $(65.7 \%$ vs $45.3, \mathrm{p}<0.001)$ and antiplatelet agents rather than anticoagulants ( $<<0.008)$, and they had more labile INR (70\% vs $53.2 \%$, p 0.018 ) but received less treatment with statins $(33.3 \%$ vs $48.5 \%$, p 0.009$)$ and suffered more stroke incidences. The outcome-independent factors were age (HR 1.02 CI95\% 1.00-1.05, p 0.048), Charlson index (HR 1.16 CI95\% 1.02-1.32, p 0.019), and HF (HR 1.83 CI95\% 1.27-2.63, p 0.001). Using a reduced model and adjusting for the strongest known predictors of mortality (age at the register of CCP, sex, and Charlson score), the Barthel score remained a significant factor in the reduction in mortality (HR 0.984 CI95\% 0.976-0.993, p <0.001).

Table 2, Figure 2

\begin{tabular}{|c|c|c|c|}
\hline CCP PEOPLE with AF (N 325) & $\begin{array}{l}\text { WITHOUT cognitive } \\
\text { impairment }\left(n_{1} 223\right)\end{array}$ & $\begin{array}{l}\text { WITH cognitive im- } \\
\text { pairment }\left(\mathrm{n}_{2} 102\right)\end{array}$ & $\mathbf{P}$ \\
\hline $\mathrm{N}(\%)$ & 223 & $102(31.38 \%)$ & \\
\hline Age (average \pm SD) & $82.8 \pm 7.90$ & $86.45 \pm 5.90$ & $<0.001$ \\
\hline Percentage $>80$ year-old $n(\%)$ & $156(70 \%)$ & $91(89.2 \%)$ & $<0.001$ \\
\hline Women n (\%) & & $58(56.9 \%)$ & 0.008 \\
\hline $\mathrm{CCP}$ criteria number (average $\pm \mathrm{SD}$ ) & $3.64 \pm 0.94$ & $4.63 \pm 1.19$ & $<0.001$ \\
\hline Charlson score (average $\pm \mathrm{SD}$ ) & $2.42 \pm 1.26$ & $3.24 \pm 1.40$ & $<0.001$ \\
\hline Hypertension n (\%) & $191(85.7 \%)$ & $83(81.4 \%)$ & 0.205 \\
\hline Diabetes mellitus n (\%) & $101(45.3 \%)$ & $48(47.1 \%)$ & 0.429 \\
\hline Dyslipidemia n (\%) & $125(56.1 \%)$ & $42(41.2 \%)$ & 0.009 \\
\hline Active smoking n (\%) & $21(9.2 \%)$ & $1(1 \%)$ & 0.002 \\
\hline
\end{tabular}




\begin{tabular}{|c|c|c|c|}
\hline CCP PEOPLE with AF (N 325) & $\begin{array}{l}\text { WITHOUT cognitive } \\
\text { impairment }\left(n_{1}, 223\right)\end{array}$ & $\begin{array}{l}\text { WITH cognitive im- } \\
\text { pairment }\left(\mathrm{n}_{2} 102\right)\end{array}$ & $\mathbf{P}$ \\
\hline Alcoholism active n (\%) & $11(4.9 \%)$ & $0(0.0 \%)$ & 0.115 \\
\hline Ischaemic Heart Disease n (\%) & $51(22.9 \%)$ & $25(24.5 \%)$ & 0.414 \\
\hline Peripheral arterial occlusive disease n (\%) & $39(17.5 \%)$ & $12(11.8 \%)$ & 0.123 \\
\hline Failure Heart n (\%) & $118(52.9 \%)$ & $54(52.9 \%)$ & 0.546 \\
\hline Daily medications number (average \pm SD) & $9.92 \pm 3.23$ & $8.83 \pm 3.36$ & 0.006 \\
\hline $\begin{array}{l}\text { CHADSVASC score } \\
\text { (average } \pm \text { SD) }\end{array}$ & $4.92 \pm 1.30$ & $5.16 \pm 1.17$ & 0.134 \\
\hline Stroke risk/year (average \pm SD) & $6.40 \pm 2.47$ & $6.87 \pm 2.27$ & 0.115 \\
\hline HAS-BLED (average \pm SD) & $2.92 \pm 1.08$ & $3.05 \pm 1.13$ & 0.339 \\
\hline Major Bleeding rate $\% /$ year (average $\pm S D$ ) & $4.76 \pm 3.48$ & $5.17 \pm 3.75$ & 0.363 \\
\hline $\begin{array}{l}\text { Oral Anticoagulant treatment } \\
\mathrm{n}(\%)\end{array}$ & $156(70.0 \%)$ & $62(60.8 \%)$ & 0.137 \\
\hline Labile INR (TTR < 60\%) & $83(53.2 \%)$ & $42(70.0 \%)$ & 0.018 \\
\hline Hypertension no controlled n (\%) & $22(9.9 \%)$ & $6(5.9 \%)$ & 0.165 \\
\hline Stroke before CCP register n (\%) & $45(20.2 \%)$ & $32(31.4 \%)$ & 0.021 \\
\hline $\begin{array}{l}\text { Stroke after CCP register n (\%) /100 } \\
\text { people/year }\end{array}$ & $\begin{array}{c}16(7.2 \%) \\
2.18 / 100 / \text { year }\end{array}$ & $\begin{array}{c}9(8.8 \%) \\
4.18 / 100 / \text { year }\end{array}$ & 0.231 \\
\hline Pfeiffer Test Score (average \pm SD) & $0.94 \pm 1.14$ & $6.03 \pm 2.08$ & $<0.001$ \\
\hline Barthel score (average \pm SD) & $77.9 \pm 24.08$ & $53.15 \pm 28$ & $<0.001$ \\
\hline $\begin{array}{c}\text { Percentage Barthel score }<60 \text { Moderate } \\
\text { dependence } \mathrm{n}(\%)\end{array}$ & $46(20.6 \%)$ & $56(54.9 \%)$ & $<0.001$ \\
\hline Gijon score (average \pm SD) & $9.89 \pm 3.34$ & $8.50 \pm 5.24$ & 0.372 \\
\hline Platelet antiaggregants n (\%) & $45(20.2 \%)$ & $34(33.3 \%)$ & 0.008 \\
\hline Statines treatment $\mathrm{n}(\%)$ & $107(48.8 \%)$ & $34(33.3 \%)$ & 0.009 \\
\hline Inhibitor proton pump n (\%) & $175(78.5 \%)$ & $74(72.5 \%)$ & 0.152 \\
\hline $\begin{array}{l}\text { Antidepressants and/or sedating or } \\
\text { similar } \mathrm{n}(\%)\end{array}$ & $101(45.3 \%)$ & $67(65.7 \%)$ & $<0.001$ \\
\hline Falls risk report n (\%) & $33(14.8 \%)$ & $21(20.6 \%)$ & 0.128 \\
\hline Death n (\%) & $75(33.6 \%)$ & $58(56.9 \%)$ & $<0.001$ \\
\hline $\begin{array}{l}\text { Average survival time (days) } \\
\quad \text { (average } \pm \mathrm{SD})\end{array}$ & $1200.4 \pm 2944.3$ & $772.0 \pm 399.0$ & 0.034 \\
\hline
\end{tabular}

Table 2: Basal characteristics Complex and Chronic Patients with Atrial Fibrillation and cognitive impairment

A total of 172 patients (52.92\% CI95\% 47.3-58.5) with AF had been diagnosed with HF. The basal characteristics are showed in

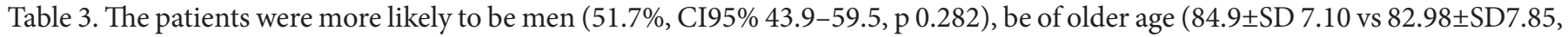
$\mathrm{p}<0.022)$, and have a higher score in the Charlson index $(2.86 \pm$ SD 1.23 vs $2.47 \pm$ SD1.45, $\mathrm{p}<0.009)$, a higher score in CHA2DS2VASC (5.28 \pm SD 1.20 vs $4.68 \pm$ SD1.26, $\mathrm{p}<0.001$ ), and higher mortality ( $50.6 \%$ vs $30.1 \%, \mathrm{p}<0.001)$ (Figure 3 ). HF remained the strongest outcome-independent factor associated with mortality after adjustment for other risk factors. HF was found to increase the mortality by two times (relative risk: 1.68 ; C.I95\%: 1.26-2.23). 


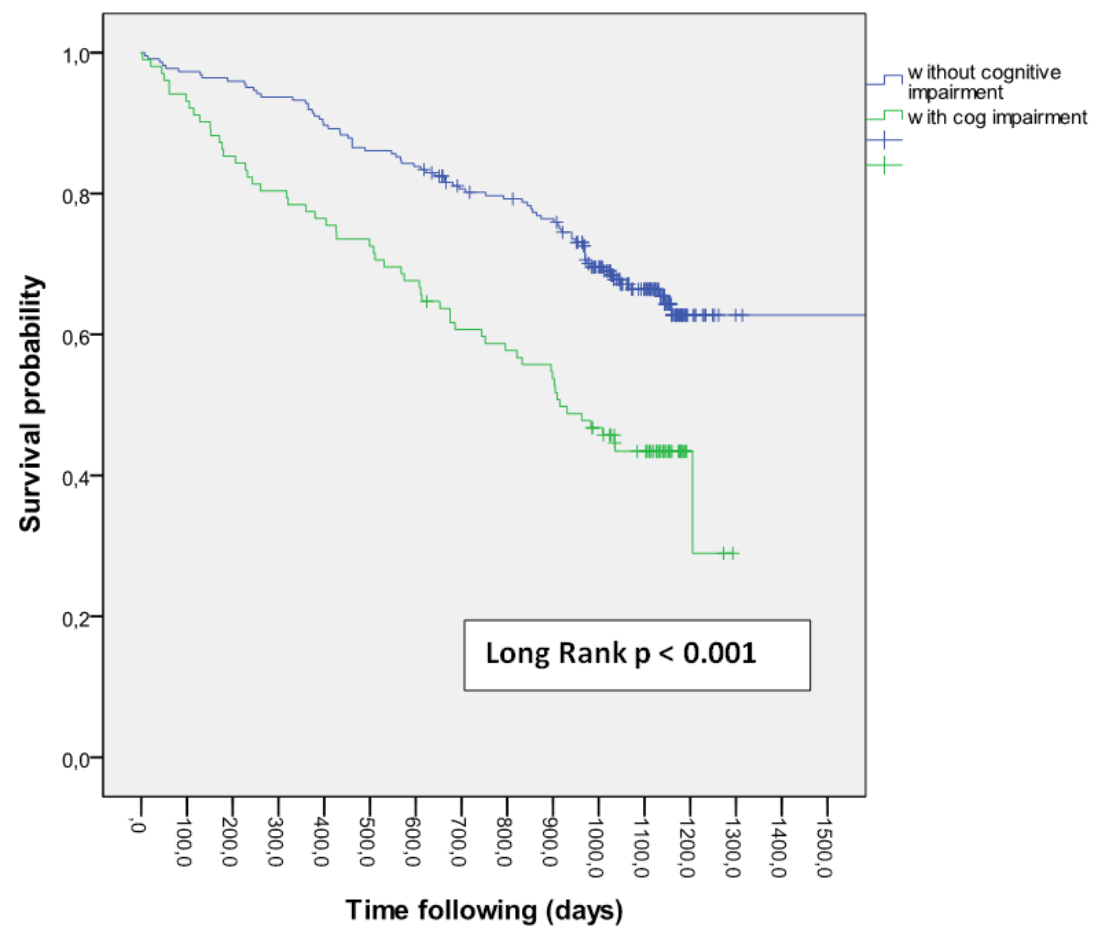

Figure 2: Survival in $\mathrm{CCP}$ with $\mathrm{AF}$ with/without cognitive impairment

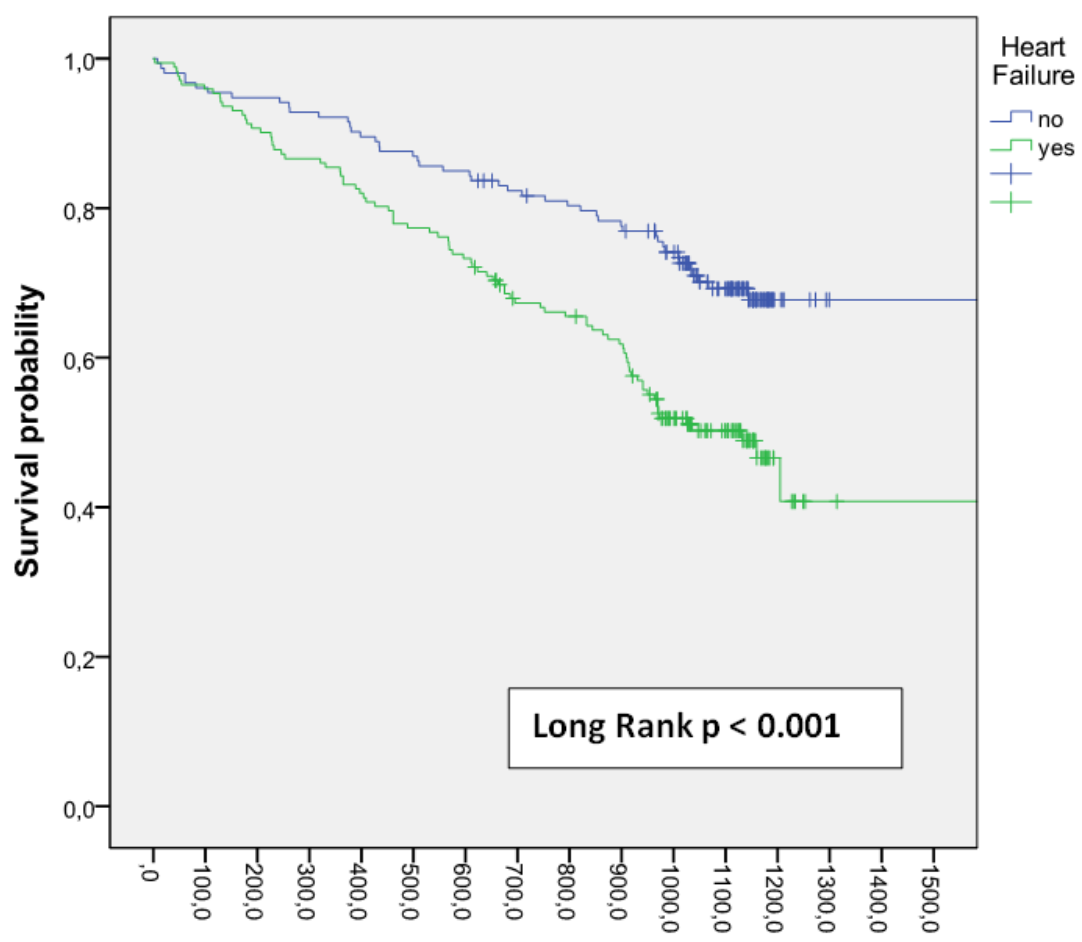

Time following (days)

Figure 3: Survival in CCP with AF and Heart failure

\begin{tabular}{|c|c|c|c|}
\hline CCP PEOPLE with AF (N 325) & $\begin{array}{c}\text { Without Heart Failure } \\
\text { (n1 153) }\end{array}$ & $\begin{array}{c}\text { With Heart Failure } \\
\text { (n2 172) }\end{array}$ & P \\
\hline N (\%) & 153 & $84.9 \pm 7.10$ & 0.022 \\
\hline Age (average \pm SD) & $82.9 \pm 7.85$ & $138(80.2 \%)$ & 0.039 \\
\hline Percentage $>80$ year-old n (\%) & $109(71.2 \%)$ & $3.99 \pm 1.04$ & 0.282 \\
\hline Women $n(\%)$ & $68(44.4 \%)$ & 0.524 \\
\hline
\end{tabular}




\begin{tabular}{|c|c|c|c|}
\hline CCP PEOPLE with AF (N 325) & $\begin{array}{l}\text { Without Heart Failure } \\
\text { (n1 153) }\end{array}$ & $\begin{array}{l}\text { With Heart Failure } \\
\text { (n2 172) }\end{array}$ & $\mathbf{P}$ \\
\hline Charlson score (average $\pm \mathrm{SD}$ ) & $2.47 \pm 1.45$ & $2.86 \pm 1.23$ & 0.009 \\
\hline Hypertension n (\%) & $133(86.9 \%)$ & $141(82.0 \%)$ & 0.142 \\
\hline Diabetes mellitus n (\%) & $78(49.7 \%)$ & $73(42.4 \%)$ & 0.116 \\
\hline Dyslipidemia n (\%) & $83(54.2 \%)$ & $84(48.8 \%)$ & 0.194 \\
\hline Active smoking n (\%) & $12(7.8 \%)$ & $10(5.8 \%)$ & 0.306 \\
\hline Alcoholism active $\mathrm{n}(\%)$ & $10(6.5 \%)$ & $1(0.6 \%)$ & 0.003 \\
\hline Ischaemic Heart Disease n (\%) & $34(22.2 \%)$ & $42(24.2 \%)$ & 0.369 \\
\hline Peripheral arterial occlusive disease $\mathrm{n}(\%)$ & $27(17.6 \%)$ & $24(14.0 \%)$ & 0.223 \\
\hline Daily medications number (average \pm SD) & $9.36 \pm 3.34$ & $9.78 \pm 3.27$ & 0.255 \\
\hline $\begin{array}{l}\text { CHADSVASC score } \\
\text { (average } \pm \text { SD })\end{array}$ & $4.68 \pm 1.26$ & $5.28 \pm 1.20$ & $<0.001$ \\
\hline Stroke risk/year (average $\pm \mathrm{SD}$ ) & $6.03 \pm 2.47$ & $7.00 \pm 2.28$ & $<0.001$ \\
\hline HAS-BLED (average \pm SD) & $2.94 \pm 1.07$ & $2.99 \pm 1.12$ & 0.687 \\
\hline Major Bleeding rate $\% /$ year (average \pm SD) & $4.80 \pm 3.59$ & $4.97 \pm 3.57$ & 0.666 \\
\hline $\begin{array}{l}\text { Oral Anticoagulant treatment } \\
\mathrm{n}(\%)\end{array}$ & $111(72.6 \%)$ & $129(74.0 \%)$ & 0.395 \\
\hline Labile INR (TTR < 60\%) & $54(55.1 \%)$ & $71(60.2 \%)$ & 0.270 \\
\hline Hypertension no controlled n (\%) & $15(9.8 \%)$ & $13(7.6 \%)$ & 0.300 \\
\hline Stroke before CCP register $\mathrm{n}(\%)$ & $40(26.1 \%)$ & $37(21.4 \%)$ & 0.198 \\
\hline $\begin{array}{l}\text { Stroke after CCP register n (\%) /100 } \\
\text { people/year }\end{array}$ & $\begin{array}{c}16(10.5 \%) \\
3.36 / 100 / \text { year }\end{array}$ & $\begin{array}{c}9(5.2 \%) \\
1.90 / 100 / \text { year }\end{array}$ & 0.134 \\
\hline Pfeiffer Test Score (average \pm SD) & $2.59 \pm 2.95$ & $2.48 \pm 2.66$ & 0.719 \\
\hline Cognitive Impairment (Peiffer score $\geq 4$ ) & $48(31.4 \%)$ & $54(31.4 \%)$ & 0.546 \\
\hline Barthel score (average $\pm \mathrm{SD}$ ) & $72.03 \pm 28.33$ & $68.55 \pm 27.86$ & 0.265 \\
\hline $\begin{array}{c}\text { Percentage Barthel score }<60 \text { Moderate } \\
\text { dependence } \mathrm{n}(\%)\end{array}$ & $43(28.1 \%)$ & $59(34.3 \%)$ & 0.140 \\
\hline Gijon score (average \pm SD) & $9.89 \pm 3.34$ & $8.50 \pm 5.24$ & 0.372 \\
\hline Platelet antiaggregants n (\%) & $40(26.1 \%)$ & $39(22.7 \%)$ & 0.275 \\
\hline Statines treatment $\mathrm{n}(\%)$ & $65(42.5 \%)$ & $76(44.2 \%)$ & 0.422 \\
\hline $\begin{array}{l}\text { Antidepressants and/or sedating or } \\
\text { similar } \mathrm{n}(\%)\end{array}$ & $76(49.7 \%)$ & $92(53.5 \%)$ & 0.282 \\
\hline Falls risk report $\mathrm{n}(\%)$ & $23(15.0 \%)$ & $31(18.0 \%)$ & 0.284 \\
\hline Death n (\%) & $46(30.1 \%)$ & $87(50.6 \%)$ & $<0.001$ \\
\hline $\begin{array}{l}\text { Average survival time (days) } \\
\text { (average } \pm \text { SD) }\end{array}$ & $1136.4 \pm 2402$ & $1003.0 \pm 2507.0$ & 0.627 \\
\hline
\end{tabular}

Table 3: Basal characteristics Complex and Chronic Patients with Atrial Fibrillation and Heart Failure.

Cognitive impairment is particularly common (31.4\%) among those with HF (Table 4) and exerts significant effects on the mortality of patients with HF (Figure 4). Among those with cognitive impairment, there was less treatment with oral anticoagulants mainly among those with a Barthel score $<60(\mathrm{p}<0.001)$ and poor quality $(\mathrm{c}-\mathrm{TTR}<60 \%)$. 


\begin{tabular}{|c|c|c|c|}
\hline CCP PEOPLE with AF (N 325) & $\begin{array}{l}\text { Atrial Fibrillation } \\
\text { (All) }\end{array}$ & $\begin{array}{c}\text { FA+Cognitive Impair- } \\
\text { ment }\end{array}$ & FA+Heart Failure \\
\hline $\mathrm{N}(\%)$ & $325(34.87 \%)$ & $102(31.38 \%)$ & $172(52.92 \%)$ \\
\hline Age (average \pm SD) & $83.9 \pm 7.51$ & $86.45 \pm 5.90$ & $84.9 \pm 7.10$ \\
\hline Percentage $>80$ year-old $n(\%)$ & $247(76.0 \%)$ & $91(89.2 \%)$ & $138(80.2 \%)$ \\
\hline Women n (\%) & $151(46.5 \%)$ & $58(56.9 \%)$ & $68(44.4 \%)$ \\
\hline $\mathrm{CCP}$ criteria number (average $\pm \mathrm{SD}$ ) & $3.95 \pm 1.12$ & $4.63 \pm 1.19$ & $3.99 \pm 1.04$ \\
\hline Charlson score (average $\pm \mathrm{SD}$ ) & $2.68 \pm 1.36$ & $3.24 \pm 1.40$ & $2.86 \pm 1.23$ \\
\hline Hypertension n (\%) & $274(84.3 \%)$ & $83(81.4 \%)$ & $141(82.0 \%)$ \\
\hline Diabetes mellitus n (\%) & $149(45.8 \%)$ & $48(47.1 \%)$ & $73(42.4 \%)$ \\
\hline Dyslipidemia n (\%) & $167(51.4 \%)$ & $42(41.2 \%)$ & $84(48.8 \%)$ \\
\hline Active smoking $\mathrm{n}(\%)$ & $22(6.8 \%)$ & $1(1 \%)$ & $10(5.8 \%)$ \\
\hline Alcoholism active n (\%) & $11(3.4 \%)$ & $0(0.0 \%)$ & $1(0.6 \%)$ \\
\hline Ischaemic Heart Disease n (\%) & $76(23.4 \%)$ & $25(24.5 \%)$ & $42(24.2 \%)$ \\
\hline Peripheral arterial occlusive disease $\mathrm{n}(\%)$ & $51(15.7 \%)$ & $12(11.8 \%)$ & $24(14.0 \%)$ \\
\hline Daily medications number (average $\pm S D$ ) & $9.58 \pm 3.31$ & $8.83 \pm 3.36$ & $9.78 \pm 3.27$ \\
\hline $\begin{array}{l}\text { CHADSVASC score } \\
\quad \text { (average } \pm \mathrm{SD})\end{array}$ & $5.00 \pm 1.26$ & $5.16 \pm 1.17$ & $5.28 \pm 1.20$ \\
\hline Stroke risk/year (average $\pm \mathrm{SD}$ ) & $6.55 \pm 2.41$ & $6.87 \pm 2.27$ & $7.00 \pm 2.28$ \\
\hline HAS-BLED (average $\pm S D$ ) & $2.96 \pm 1.09$ & $3.05 \pm 1.13$ & $2.99 \pm 1.12$ \\
\hline Major Bleeding rate $\% /$ year (average $\pm S D$ ) & $4.89 \pm 3.57$ & $5.17 \pm 3.75$ & $4.97 \pm 3.57$ \\
\hline $\begin{array}{l}\text { Oral Anticoagulant treatment } \\
\mathrm{n}(\%)\end{array}$ & $240(73.9 \%)$ & $62(60.8 \%)$ & $129(74.0 \%)$ \\
\hline Labile INR (TTR < 60\%) & $125(57.9 \%)$ & $42(70.0 \%)$ & $71(60.2 \%)$ \\
\hline Hypertension no controlled n (\%) & $28(8.6 \%)$ & $6(5.9 \%)$ & $13(7.6 \%)$ \\
\hline Stroke before CCP register $\mathrm{n}(\%)$ & $\begin{array}{c}77(23.7 \%) \\
8.11 / 100 / \text { year }\end{array}$ & $\begin{array}{c}32(31.4 \%) \\
14.86 / 100 / \text { year }\end{array}$ & $\begin{array}{l}37(21.4 \%) \\
7.85 / 100 / \text { year }\end{array}$ \\
\hline $\begin{array}{l}\text { Stroke after CCP register n (\%) /100 } \\
\text { people/year }\end{array}$ & $\begin{array}{c}25(7.7 \%) \\
2.63 / 100 / \text { year }\end{array}$ & $\begin{array}{c}9(8.8 \%) \\
4.18 / 100 / \text { year }\end{array}$ & $\begin{array}{c}9(5.2 \%) \\
1.90 / 100 / \text { year }\end{array}$ \\
\hline Pfeiffer Test Score (average \pm SD) & $2.54 \pm 2.80$ & $6.03 \pm 2.08$ & $2.48 \pm 2.66$ \\
\hline Barthel score (average $\pm \mathrm{SD}$ ) & $70.2 \pm 28.09$ & $53.15 \pm 28$ & $68.55 \pm 27.86$ \\
\hline $\begin{array}{l}\text { Percentage Barthel score }<60 \text { Moderate } \\
\text { dependence } \mathrm{n}(\%)\end{array}$ & $102(31.4 \%)$ & $56(54.9 \%)$ & $59(34.3 \%)$ \\
\hline Gijon score (average $\pm \mathrm{SD}$ ) & $9.35 \pm 4.16$ & $8.50 \pm 5.24$ & $8.50 \pm 5.24$ \\
\hline Platelet antiaggregants n (\%) & $79(24.3 \%)$ & $34(33.3 \%)$ & $39(22.7 \%)$ \\
\hline Statines treatment $\mathrm{n}(\%)$ & $23(35.4 \%)$ & $74(72.5 \%)$ & $76(44.2 \%)$ \\
\hline $\begin{array}{l}\text { Antidepressants and/or sedating or } \\
\text { similar } \mathrm{n}(\%)\end{array}$ & $168(51.7 \%)$ & $67(65.7 \%)$ & $92(53.5 \%)$ \\
\hline Falls risk report n (\%) & $54(16.6 \%)$ & $21(20.6 \%)$ & $31(18.0 \%)$ \\
\hline Death n (\%) & $133(40.9 \%)$ & $58(56.9 \%)$ & $87(50.6 \%)$ \\
\hline $\begin{array}{c}\text { Average survival time (days) } \\
\text { (average } \pm S D)\end{array}$ & $1066.01 \pm 2455.4$ & $772.0 \pm 399.0$ & $1003.0 \pm 2507.0$ \\
\hline
\end{tabular}

Table 4: Differential characteristics of CCP people with Atrial Fibrillation and Cognitive Impairment 


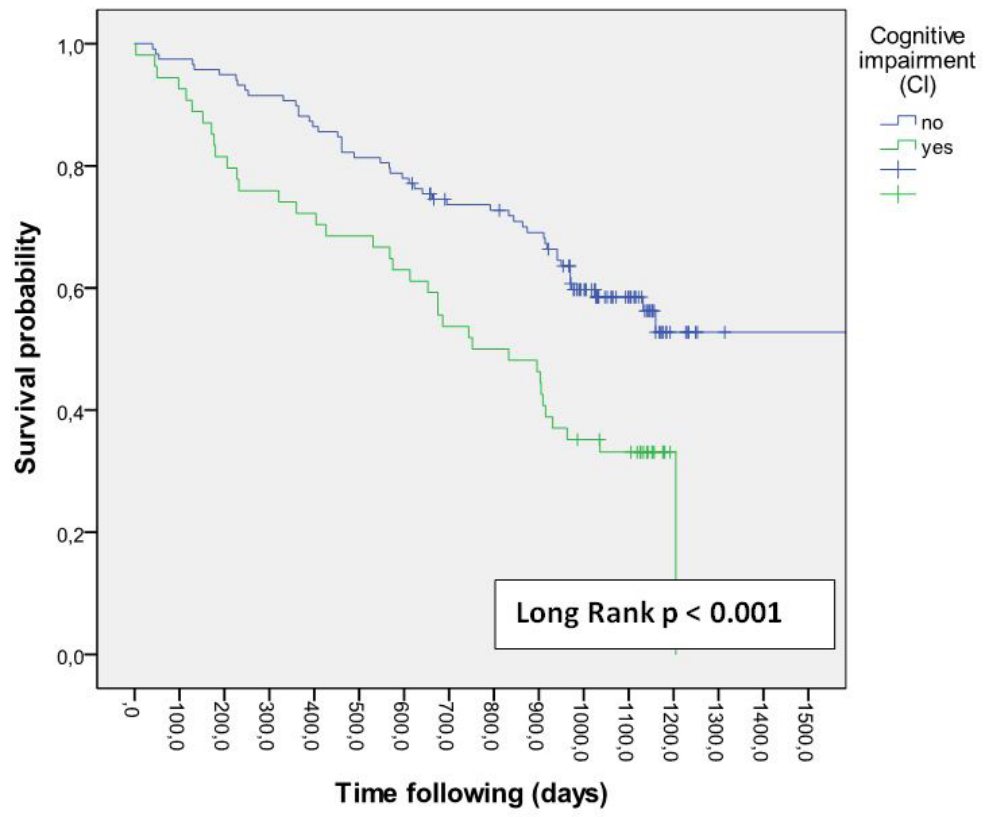

Figure 4: Survival in [AF and heart failure] with cognitive impairment

The overall mortality among the CCP with AF was $40.9 \%$ and $56.9 \%$ if associated with cognitive impairment ( $\mathrm{p} 0.001$ ). The longterm survival was not different between the groups of CCP with AF and without AF (p 0.463). In the unadjusted analysis, patients who had AF were at a significantly higher risk of death if they were $\geq 80$ years old, had cognitive impairment, or had a Barthel score $<60$.

These patients also had the worse results in the quality of anticoagulant treatment. Therefore, persons with AF should be investigated for the presence of cognitive impairment and HF, given their higher prevalence and prognostic importance, which should be a major priority in the treatment of $\mathrm{AF}$, as the impact of anticoagulation on long-term cognitive function.

\section{Discussion}

The present research is one of the few prospective studies on demographic outbreak of atrial fibrillation, cognitive deterioration, and heart failure that assessed the vital prognosis in individuals considered complex chronic patients. In terms of their relation to the health system, CCPs are characterized by their recurrent use of health services (primary, specialized, and emergency care) and by multiple hospital admissions, in some cases for long stays. Chronic complex patients require care from a combination of professionals, need to undergo numerous diagnostic tests, and are prescribed a range of drugs. They have low adherence to treatment, and the drugs may have side effects or interact. In addition, patients' social and economic situation has a great impact on how the disease evolves. These patients are intensive users of health resources and constitute an important cause of the increase in healthcare costs. In addition, the increase in the number of associated comorbidities causes a multiplier effect rather than additive effects in the structural costs. These facts suggest that the management and coordination of healthcare programmes will face the added challenge of heterogeneity in the health status of this population group [20].

The meaning of the concept 'complex chronic patient' is similar to that of 'fragility'. It is a new concept that has not yet been well defined nor has it gained consensus in the literature [21]. Fragility is a complex issue that encompasses a set of clinical conditions, such as: limited mobility, with a high associated risk of falls; polypharmacy; others comorbidities; high social risk; nutritional problems; and cognitive deterioration.

The significant incidence of atrial fibrillation in individuals with cognitive deterioration compared to those without it, as well as the association between cardiovascular diseases and cognitive deterioration are well defined [22-26]. Our findings add a strong association between mortality risk and the presence of atrial fibrillation and/or cognitive deterioration and/or heart failure. Various studies have demonstrated that cognitive deterioration is particularly common in 30 to $80 \%$ of patients with heart failure [27]. Cognitive deterioration can interfere with the ability to perform self-care activities, and can be associated with increased mortality in this group of patients [28].

In addition, adherence to treatment can be significantly undermined by the difficulty of patients in making appropriate decisions with respect to their needs. This fact may have significant consequences in terms of patients' quality of life, associated disabilities, and control of associated comorbidities and mortality. Cognitive deterioration can also generate or worsen social and behavioural problems. This way, the challenge for health professionals would not only be the treatment of heart failure, but also the identification and treatment of associated conditions - such as cognitive deterioration - in order to avoid further complications. An integral clinical management of these conditions provided by the community nursing service could improve the adhesion to clinical guidelines, promote patient participation, and efficiently reduce the complications of chronic comorbidity and mortality [29]. 
Therefore, one of the relevant priorities in the management of atrial fibrillation is that individuals with this disorder should be systematically assessed to detect the occurrence of cognitive deterioration and heart failure. Health professionals should be familiar with early detection of cognitive symptoms in their daily routine. Even though the most effective treatment to prevent cognitive deterioration associated with atrial fibrillation is currently unknown, the patients should be encouraged to participate in cognitive, physical, and social activities with the purpose of improving and/or preserving their cognitive skills.

There are no data relating to the benefits of oral anticoagulants. According to the clinical guidelines, if the time in therapeutic range (TTR) cannot be maintained at $\geq 60 \%$ with the use of anti-vitamin $\mathrm{K}$, the use of new oral anticoagulants (NOACs) should be taken into consideration [1]. Internationally, qualitative studies on the treatment with anti-vitamin $\mathrm{K}$ have shown poor results in the general practice and untreated patients are associated with atrial fibrillation burden, chronicity, and comorbidity for adverse outcomes [7,30-31]. In the present study, we observed that $70 \%$ of the patients with cognitive deterioration had a TTR of $<60 \%$, as well as $60 \%$ of patients with heart failure. This way, cognitive decline associated with poor results in TTR again suggests that there may be a relationship between the two conditions $[31,32]$.

However, further long-term studies are necessary to obtain evidence about the effect of TTR and the use of NOACs for the evolutionary diagnosis of the association between cognitive deterioration and atrial fibrillation, as well as the effects on heart failure.

Even though the $\mathrm{CHA}_{2} \mathrm{DS}_{2}-\mathrm{VAS}_{\mathrm{C}}$ and HAS-BLED score values progressively increase with age until 85 years, the percentage of patients treated with oral anticoagulants is not modified or even decreases in the group of patients with cognitive deterioration. This fact may result from the decisions made by health professionals with respect to the risk of bleeding and the risk-benefit of the treatment in high-risk populations, as is the case of the group of complex chronic patients.

The deficit in the use of anticoagulants in this population is evident and the reasons are little known, although it has been suggested that the clinical criteria used to prescribe oral anticoagulants are different from those indicated by risk scales [7,33]. Despite the clear indication of anticoagulant treatment according to the risk scales, in the daily practice this treatment is often interrupted in complex chronic patients with atrial fibrillation, even though it could contribute to a lower cerebrovascular morbidity and mortality rate [30].

Although the assessment of complex chronic patients using the CHA2DS2-VASC and HAS-BLED scales can facilitate determining the vital prognosis in those patients with atrial fibrillation, our results only showed this association in those patients with heart failure [34]. Finally, some studies have provided evidence about which specific medication-such as angiotensin-converting enzyme inhibitors and digoxin-may have beneficial effects on the cognitive performance of patients with heart failure [35].

The results confirm that cognitive deterioration in complex chronic patients with atrial fibrillation is associated with a greater mortality risk. These patients are older, suffer from more chronic diseases, and have a worse functional status [9]. In addition, isolation and loneliness have been described as concomitant risk factors. In the present study, the percentage of loneliness was $22.3 \%$ among those patients aged $\geq 75$ years. Since these factors can worsen the condition of complex chronic patients, those without care and/or social support should be considered a group of high priority for preventive interventions provided by community nursing.

We believe that the model of chronic care, in which our proposal is based, should be characterised by integral intervention aimed at: (a) stimulating the cognitive condition of the patients; (b) avoiding their social isolation; (c) detecting symptoms and risk situations; (d) achieving objectives of control according to the clinical guidelines for the effective control of cardiovascular risk factors; and (e) achieving therapeutic objectives through the prescription of oral anticoagulants. It is worth mentioning that this intervention should be carried out by a multidisciplinary team working with a shared clinical record led by nursing professionals. This strategy should improve their quality of treatment and life. In addition, as patients are evaluated continuously, they feel better protected. Eventually, the system might avoid institutionalization and use of repeated and probably unnecessary trips to the hospital, time in emergency rooms, and give a great support to the patient's caretaker and family.

\section{Conclusion}

We concluded two main facts: the high prevalence of heart failure (52.9\%) and cognitive deterioration (31.4\%) associated to lower long-term survival rate; and, the poor results in the quality of anticoagulant treatment among the patients with atrial fibrillation, cognitive deterioration and heart failure.

Therefore, persons with AF should be investigated for the presence of cognitive impairment and heart failure and should be independently assessed the impact of quality and kind of anticoagulation on the long-term survival in future research.

\section{References}

1. European Heart Rhythm Association, European Association for Cardio-Thoracic Surgery, Camm AJ, Kirchhof P, Lip GY, et al. (2010) Guidelines for the management of atrial fibrillation: the task force for the management of atrial fibrillation of the European Society of Cardiology (ESC). Eur Heart J 31: $2369-429$.

2. Stefansdottir H, Aspelund T, Gudnason V, Arnar DO (2011) Trends in the incidence and prevalence of atrial fibrillation in Iceland and future projections. Europace 13: 1110-7. 
3. Miyasaka Y, Marion E. Barnes, Bernard J. Gersh, Stephen S, et al. (2006) Secular trends in incidence of atrial fibrillation in Olmsted County, Minnesota, 1980 to 2000, and implications on the projections for future prevalence. Circulation 114: 119-25.

4. Hui DS, Morley JE, Mikolajczak PC, Lee R (2015) Atrial fibrillation: a major risk factor for cognitive decline. Am Heart J 169: $448-456$.

5. Kwok CS, Loke YK, Hale R, Potter JF, Myint PK (2011) Atrial fibrillation and incidence of dementia: a systematic review and meta-analysis. Neurology 76: 91422.

6. Cao L, Pokorney SD, Hayden K, Welsh-Bohmer K, Newby LK (2015) Cognitive function: is there more to anticoagulation in atrial fibrillation than stroke? J Am Heart Assoc. 4: e001573. doi: 10.1161/JAHA.114.001573

7. Panisello-Tafalla A, Lluís Clua-Espuny J, Gil-Guillen VF, González-Henares A, Queralt-Tomas ML, et al. (2015) Results from the Registry of Atrial Fibrillation (AFABE): gap between undiagnosed and registered atrial fibrillation in adults-ineffectiveness of oral anticoagulation treatment with VKA. BioMed Research International.

8. Petersen RC (2011) Clinical practice. Mild cognitive impairment. N Engl J Med. 364: 2227-34.

9. Alzheimer's Disease International (2013) Policy brief for G8 heads of government. The global impact of dementia 2013-2050. Alzheimer's Disease International, London.

10. World Health Organization (2012) Dementia: A public health priority. World Health Organization, Geneva.

11. Harmsen P, Wilhelmsen L, Jacobsson A (2009) Stroke incidence and mortality rates 1987 to 2006 related to secular trends of cardiovascular risk factors in Gothenburg, Sweden. Stroke 40: 2691-7.

12. Wodchis WP, Bhatia RS, Leblanc K, Meshkat N, Morra D (2012) A review of the cost of atrial fibrillation. Value in Health 15: $240-8$.

13. Josep Lluís Clua-Espuny, Ramon Bosch-Princep, Albert Roso-Llorach, Carlos López-Pablo, Emmanuel Giménez-Garcia, Núria González-Rojas, et al. (2014) Diagnosed, undiagnosed and overall atrial fibrillation research on population over 60 year-old. AFABE study. Cardiovascular System 2.

14. Gunn J, Naccarella L, Palmer V, Kokanovic R, Pope C, Lathlean J (2007) What is the place of generalism in the 2020 primary care team? Australian Primary Health Care Institute, Melbourne.

15. Catalunya. Departament de Salut. (2013) Terminologia de la cronicitat [en línia]. TERMCAT, Centre de Terminologia, Barcelona. (Diccionaris en Línia).

16. Charlson M, Pompei P, Ales KL, McKenzie CR (1987) A new method of classifying prognostic comorbidity in longitudinal studies: development and validation. J Chron Dis 40: 373-83.

17. Pfeiffer E (1975) A short portable mental status questionnaire for the assessment of organic brain deficit in elderly patients. J Am Geriatr Soc. 23: 433-41.

18. Shamsham F, Mitchell J (2000) Essentials of the diagnosis of heart failure. Am Fam Physician 61:1319-28.

19. García-González JV, Díaz-Palacios E, Salamea A, Cabrera D, Menéndez A, et al. (1999) Evaluación de la fiabilidad y validez de una escala de valoración social en el anciano. Aten Primaria. 23: 434-40.

20. Sorace J, Wong HH, Worrall C, Kelman J, Saneinejad S, et al. (2011) The complexity of disease combinations in the Medicare population. Population Health Management 14: 161-6.

21. Qian-Li X (2011) The frailty syndrome: definition and natural history. Clin Geriatr Med. 27: 1-15.

22. Ott A, Breteler MM, de Bruyne MC, van Harskamp F, Grobbee DE, et al. (1997) Atrial fibrillation and dementia in a population-based study. The Rotterdam Study. Stroke 28: 316-21.

23. Vogels RL, Scheltens P, Schroeder-Tanka JM, Weinstein HC (2007) Cognitive impairment in heart failure: a systematic review of the literature. Eur J Heart Fail 9: 440-49.

24. Thacker EL, McKnight B, Psaty BM, Longstreth WT Jr, Sitlani CM, et al. (2013) Atrial fibrillation and cognitive decline: a longitudinal cohort study. Neurology 81: 119-25.

25. Horstmann S, Rizos T, Rauch G, Fuchs M, Arden C, et al. (2014) Atrial fibrillation and prestroke cognitive impairment in stroke. J Neurol. $261: 546-53$.

26. Dublin S, Anderson ML, Haneuse SJ, Heckbert SR, Crane PK, et al. (2011) Atrial fibrillation and risk of dementia: a prospective cohort study. J Am Geriatr Soc. 59: 1369-75.

27. Vogels RLC, Scheltens P, Schroeder-Tanka JM, Weinstein HC (2007) Cognitive impairment in heart failure: a systematic review of the literature. European Journal of Heart Failure 9: 440-9.

28. Davis KK1, Himmelfarb CR, Szanton SL, Hayat MJ, Allen JK (2015) Predictors of heart failure self-care in patients who screened positive for mild cognitive impairment. J Cardiovasc Nurs. 30: 152-60.

29. Hendriks JML, de Wit R, Crijns HJGM, Vrijhoef HJM, Prins MH, et al. (2012) Nurse-led care vs. usual care for patients with atrial fibrillation: results of a randomized trial of integrated chronic care vs. routine clinical care in ambulatory patients with atrial fibrillation. European Heart Journal 33: $2692-9$.

30. Ansell J, Hollowell J, Pengo V, Martinez-Brotons F, Caro J, et al. (2007) Descriptive analysis of the process and quality of oral anticoagulation management in real-life practice in patients with chronic non-valvular atrial fibrillation: the International Study of Anticoagulation Management (ISAM). Journal of Thrombosis and Thrombolysis 23: 83-91.

31. Clua-Espuny JL, Lechuga-Duran I, Bosch-Princep R, Roso-Llorach A, Panisello-Tafalla A, et al. (2013) Prevalencia de la fibrilación auricular desconocida y la no tratada con anticoagulantes. Estudio AFABE. Rev Esp Cardiol. 66: 545-52.

32. Flaker GC, Pogue J, Yusuf S, Pfeffer MA, Goldhaber SZ, et al. (2010) Cognitive function and anticoagulation control in patients with atrial fibrillation. Circ Cardiovasc Qual Outcomes 3: 277-83.

33. Bo M, Sciarrillo I, Maggiani G, Falcone Y, Iacovino M, et al. (2016) Health status, geriatric syndromes and prescription of oral anticoagulant therapy in elderly medical inpatients with atrial fibrillation. Geriatr Gerontol Int. 17: 416-23.

34. Kim SW, Yoon SJ, Choi JY, Kang MG, Kim KI, et al. (2016) Frailty assessment in older atrial fibrillation patients. J Hypertens. 34(Suppl 1): e250.

35. Laudisio A, Marzetti E, Pagano F, Cocchi A, Bernabei R, et al. (2009) Digoxin and cognitive performance in patients with heart failure: a cohort, pharmacoepidemiological survey. Drugs and Aging 26: 103-12. 


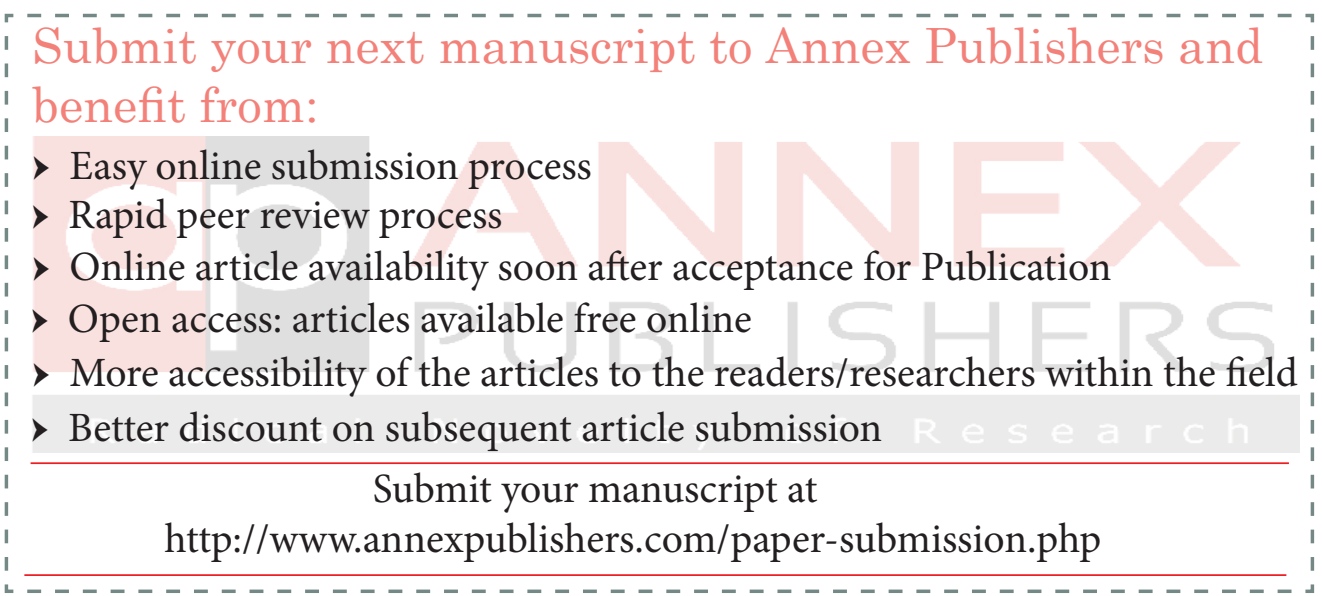

